\title{
Superscaling analysis of the Coulomb sum rule in quasielastic electron-nucleus scattering
}

\author{
J.A. Caballero $^{\mathrm{a}, *}$, M.C. Martínez $^{\text {b }}$, J.L. Herraíz ${ }^{\mathrm{b}}$, J.M. Udías ${ }^{\mathrm{b}}$ \\ a Departamento de Física Atómica, Molecular y Nuclear, Universidad de Sevilla, 41080 Sevilla, Spain

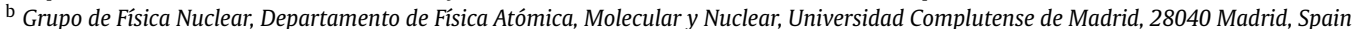

\section{A R T I C L E I N F O}

\section{Article history:}

Received 22 December 2009

Received in revised form 18 March 2010

Accepted 25 March 2010

Available online 2 April 2010

Editor: W. Haxton

\section{Keywords:}

Inclusive quasielastic electron scattering

Scaling

Superscaling

Relativistic mean field

Coulomb sum rule

\begin{abstract}
A B S T R A C T
The Coulomb sum rule for inclusive quasielastic electron scattering in ${ }^{12} \mathrm{C},{ }^{40} \mathrm{Ca}$ and ${ }^{56} \mathrm{Fe}$ is analyzed based on scaling and superscaling properties. Results obtained in the relativistic impulse approximation with various descriptions of the final state interactions are shown. A comparison with experimental data measured at Bates and Saclay is provided. The theoretical description based on strong scalar and vector terms present in the relativistic mean field, which has been shown to reproduce the experimental asymmetric superscaling function, leads to results that are in fair agreement with Bates data while it sizeably overestimates Saclay data. We find that the Coulomb sum rule for a momentum transfer $q \geqslant 500 \mathrm{MeV} / c$ saturates to a value close to 0.9 , being very similar for the three nuclear systems considered. This is in accordance with Bates data, which indicates that these show no significative quenching in the longitudinal response.
\end{abstract}

(c) 2010 Elsevier B.V. All rights reserved.

\section{Introduction}

Strongly correlated many-body systems are of interest in very diverse areas of physics. In particular, nuclei have been explored in depth by means of electron scattering reactions for very different kinematical situations. More than 50 years of experimentation have proved that electron scattering provides one of the best tools for investigating the structure of nuclear systems and their constituents [1-5]. The electromagnetic interaction with which electrons probe nuclei is well under control and is weak enough so that the process can be treated in first order photon exchange. Under this assumption (Born Approximation), it is possible to isolate the different components of the nuclear response by changing appropriately the electron kinematical variables. Indeed, assuming the Plane Wave Born Approximation (PWBA), i.e., only one virtual photon exchanged and electrons described as free particles, the inclusive $\left(e, e^{\prime}\right)$ quasielastic $(\mathrm{QE})$ differential cross section is written as [1-3],

$\frac{d \sigma}{d \varepsilon^{\prime} d \Omega^{\prime}}=\sigma_{M}\left[v_{L} R^{L}(q, \omega)+v_{T} R^{T}(q, \omega)\right]$,

where $\left(\varepsilon^{\prime}, \Omega^{\prime}\right)$ are the energy and solid angle of the scattered electron, $\sigma_{M}$ is the Mott cross section, and $v_{L}\left(v_{T}\right)$ the longitu-

\footnotetext{
* Corresponding author.

E-mail address: jac@us.es (J.A. Caballero).
}

dinal (transverse) leptonic kinematic factors that in the extreme relativistic limit (ERL) for the electrons are simply given as $v_{L}=$ $\left(Q^{2} / q^{2}\right)^{2}$ and $v_{T}=\tan ^{2}\left(\theta_{e} / 2\right)-Q^{2} / 2 q^{2}$ with $\theta_{e}$ the electron scattering angle and $(\omega, q)$ the energy and momentum transferred in the process $\left(Q^{2}=\omega^{2}-q^{2}\right)$. The hadronic $R^{K}(q, \omega)$ response functions are constructed from the nuclear electromagnetic tensor $W^{\mu \nu}$ given in terms of the initial and final many-body nuclear state, and the nuclear electromagnetic many-body current operator [2-5].

In the case of $\mathrm{QE}$ kinematics and the momentum of the exchanged photon large enough (its wavelength being of the order of or smaller than the nucleon size), knockout of a single nucleon is the dominant contribution to the nuclear response. Under these conditions, the impulse approximation (IA) holds, and the inclusive $\left(e, e^{\prime}\right)$ cross section can be given as the integrated semi-inclusive single-nucleon knockout cross sections. This approximation, which is implicit in scaling analyses, has been shown to work properly in the kinematic region dominated by the QE process [6-10].

Lowest nucleon resonances are mainly excited with purely transverse photons, hence they do not affect the longitudinal response which essentially captures the purely nucleonic contribution to the nuclear response. Assuming that the nucleons are the only relevant degrees of freedom, sum rules have been derived in both relativistic [6] and nonrelativistic schemes [11]. These sum rules can be stated separately for the longitudinal and the transverse contributions to the inclusive cross section. In particular, the 
Coulomb sum rule (CSR) states that by integrating the longitudinal strength over the full range of energy loss $\omega$ at large enough momentum transfer $q$, one should get the total charge (number of protons) of the nucleus. While the experimental realization of the transverse sum rule gets contributions from resonance excitations and thus, will likely be above theoretical estimates based only upon nucleonic degrees of freedom, the experimental CSR is suitable to comparison with theoretical predictions. Not only the asymptotic value of the CSR for large $q$, but also the evolution of the CSR with increasing $q$, is of interest in order to test nuclear models and/or descriptions of the reaction mechanism.

Indeed, enormous experimental efforts have been made at different laboratories, Saclay [12-14], Bates [15], JLAB [16], to get separated longitudinal and transverse contributions from QE electron scattering data. The analysis of data and its impact on the CSR for different nuclei have been discussed in the literature, leading to different conclusions concerning the role played by several ingredients: nucleon correlations, final state interactions, modification of the nucleon form factors by the nuclear medium, etc. Jourdan concluded that the integrated longitudinal $(L)$ response function saturates for $q$ high enough at the 100\% of the CSR limit [7], and thus it is not suppressed, showing no A-dependent quenching. On the contrary, from the analysis of data taken at Saclay [12], Morgenstern and Meziani have concluded the existence of a significant quenching of the CSR, and have interpreted such suppression as due to the change of the nucleon properties inside the nuclear medium [14]. Being aware of the present controversy, the most comprehensive effort to measure separated longitudinal inclusive responses from several nuclei and different values of momentum transfer $q$, in a large enough range of energy and with unprecedent high statistics and small systematic errors, has been recently completed at JLAB [16]. This experiment is currently under analysis and preliminary results will be released in the next months.

An alternative procedure to get some insight into the CSR relies on the information provided by the scaling properties of the longitudinal separated data. As already shown in previous works [17-19], world $\left(e, e^{\prime}\right)$ data have clearly demonstrated the validity of scaling and superscaling (independence of the scaled response on the kinematics and on the nuclear target) behavior. In particular, the analysis of the separated $L$ contribution has led to introduce a "universal" superscaling function, which contains the relevant information about the initial and final state nuclear dynamics explored by the probe [19]. Superscaling was originally introduced within the simple Relativistic Fermi Gas (RFG) model that, albeit showing perfect scaling and superscaling properties, yields a superscaled function shape not in accordance with data $[19,20]$.

The experimental superscaling function has an asymmetric shape, with a long tail exhibiting strength for energy transfers well beyond the RFG domain. Further, most nonrelativistic models also lack the significative strength at high- $\omega$ [21]. The presence of this tail is of relevance for the CSR analysis, as the sum rule requires integration of the strength in the whole energy transfer range (up to infinity), which is of course not feasible from the experimental point of view. The integration of the experimental strength ends at some finite value of the transferred energy, located where the asymmetric tail of the superscaling function resides. Thus, what is left out of the integration region from theoretical estimates of the CSR would highly depend on whether the model does or does not reproduce this asymmetric tail. The main aim of this work is trying to shed some light to the CSR problem, making use of the experience acquired during the analysis of the scaling and superscaling phenomenon $[18,19]$.

\section{Scaling and superscaling}

The usual procedure to get the scaling function consists in dividing the inclusive differential cross section (1) by the appropriate single-nucleon eN elastic cross section, weighted by the corresponding proton and neutron numbers $[18,19,22]$ involved in the process,

$f\left(\psi^{\prime}, q\right) \equiv k_{F} \frac{\left[\frac{d \sigma}{d \varepsilon^{\prime} d \Omega^{\prime}}\right]}{\sigma_{M}\left[V_{L} G_{L}(q, \omega)+V_{T} G_{T}(q, \omega)\right]}$.

$\psi^{\prime}(q, \omega)$ is the dimensionless scaling variable extracted from the RFG analysis that incorporates the typical momentum scale for the selected nucleus [19]. The fully relativistic expressions for $G_{L(T)}$ involve proton and neutron form factors $G_{E(M) p n}$, weighted by proton and neutron numbers, and an additional dependence on the nuclear scale given through the Fermi momentum $k_{F}$ (explicit expressions are given by Eqs. (16)-(19) in [19]). Analogously, the analysis of the separated longitudinal $(L)$ and transverse $(T)$ contributions leads to scaling functions,

$f_{L(T)}\left(\psi^{\prime}, q\right) \equiv k_{F} \frac{R^{L(T)}(q, \omega)}{G_{L(T)}(q, \omega)}$.

At transferred energies above the $\mathrm{QE}$ peak, scaling is violated in the transverse channel by effects beyond the impulse approximation $[17,18]$. However, the available data for the $L$ response are compatible with scaling in all the $\mathrm{QE}$ region. This has made it possible to extract an experimental scaling function $f_{L}^{\exp }$, that effectively represents the nucleon contribution to the nuclear response under QE kinematics [18,19]. In this work we use the Relativistic Impulse Approximation (RIA) that leads to a hadronic tensor evaluated from the transition single-nucleon current matrix elements. These are constructed making use of the relativistic bound-state, the scattering wave function and the relativistic single-nucleon electromagnetic current operator. We guide our analysis with calculations where the bound nucleon states correspond to self-consistent Dirac-Hartree solutions, derived within a relativistic mean field (RMF) approach. The outgoing nucleon wave function is given as a solution of the Dirac equation in presence of a relativistic potential which takes care of the final state interactions (FSI) between the ejected nucleon and the residual nucleus. In previous works [23-25] it has been investigated the role played by different descriptions of FSI: (i) the use of the same relativistic mean field employed to describe the initial bound states and (ii) considering the phenomenological relativistic optical potential derived by Clark et al. [26] but with their imaginary part set to zero in order to consider all final channels and not only the elastic one, that we denoted as rROP. Finally, ignoring all distortion from FSI leads to the relativistic plane wave impulse approximation (RPWIA) where the knocked out nucleon is treated as a plane wave.

In recent works $[23,24,27]$ it has been shown that the RMF model, where the same relativistic potentials are applied to the initial and the final state, reproduces satisfactorily the magnitude and detailed shape of $f_{L}^{\text {exp }}$, while other models fail to reproduce the long tail appearing at high energy transfer $\omega$ (large positive values of the scaling variable $\psi^{\prime}$ ). This is clearly illustrated in Fig. 1 where we present the superscaling function evaluated within the RIA and different descriptions of FSI. Results correspond to ${ }^{12} \mathrm{C}$ and $q=700 \mathrm{MeV} / c$. The scaling function obtained using the real part of the relativistic optical potential (rROP) is compared to the plane wave limit (RPWIA) and to results obtained in presence of the scalar and vector terms in the relativistic mean field potential (RMF), where the separate $L$ and $T$ contributions to the scaling function are plotted. Scaling of zeroth kind, i.e., $f_{L}=f_{T}=f$, has 


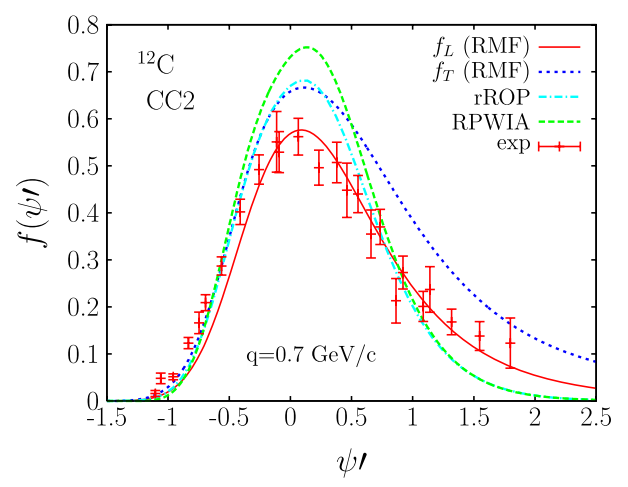

Fig. 1. (Color online.) Superscaling function for ${ }^{12} \mathrm{C}\left(e, e^{\prime}\right)$ evaluated with the RPWIA, rROP and RMF approaches compared to the experimental function. In the RMF case, separate $L$ and $T$ contributions are shown.

been shown to be fulfilled within RPWIA and rROP approaches $[24,28]$. In all the cases, the CC2 prescription for the current operator has been selected [24]. As observed, $f_{T}$ obtained within RMF is increased with regard to $f_{L}$. This is due to the off-shellness of the nucleons, modest for RPWIA and rROP and much more important for RMF because of the stronger potentials involved in the final nucleon states. While the function $f_{L}$ hardly changes (a consequence of current conservation), $f_{T}$ exhibits a significant dependence with off-shell nucleon effects [24,28].

It is worth mentioning that correlations also shift strength towards larger energy values, as they allow for multi-nucleon knockout. Correlations have been a common ingredient of theoretical predictions of CSR [29-31] and can also explain the asymmetrical shape of the superscaling function [32]. In this work our focus is not the explanation of the observed asymmetry, that has been discussed in previous work $[23,24,27,28]$, but rather explore its effect on the predicted CSR values. The comparison with the experimental $L$ superscaling function, also provided in Fig. 1, shows that the RMF approach follows closely the behavior of data describing also the asymmetrical shape of $f_{L}^{\exp }$.

Moreover, the RMF model, as studied in previous work [24,28], fullfills the continuity equation and dispersion relations, hence being adequate to inclusive scattering where all nucleon propagation channels, not only the elastic one described by the optical potentials, must be incorporated. The different behavior presented by RMF and rROP (Fig. 1) is linked directly to the strong potentials present in the RMF for large values of the energy transfer. On the contrary, rROP potentials tend to weaken significantly with increasing energy values. This is also consistent with the similar behavior shown by rROP and RPWIA results. The potentials modify the effective values of the momenta at the nucleon vertex, giving rise to a shift of strength to (asymptotical) larger values of $\omega$ (see Refs. [23,24,27,28]). Finally, use of relativistic optical potentials (with imaginary term) in inclusive reactions has been applied within the relativistic Green's function (GF) approach, leading to similar results to RMF, i.e., with presence of the asymmetry in the scaling function for intermediate $q$-values [33].

\section{The Coulomb sum rule}

Including relativistic corrections [34] and the structure of the nucleons, the explicit expression for the CSR, widely used by experimentalists in the analysis of the separate L-data [12,15], is written as

$\operatorname{CSR}(q)=\frac{1}{Z} \int_{\omega^{+}}^{\infty} \frac{R^{L}(q, \omega)}{\widetilde{G}_{E}^{2}\left(Q^{2}\right)} d \omega$
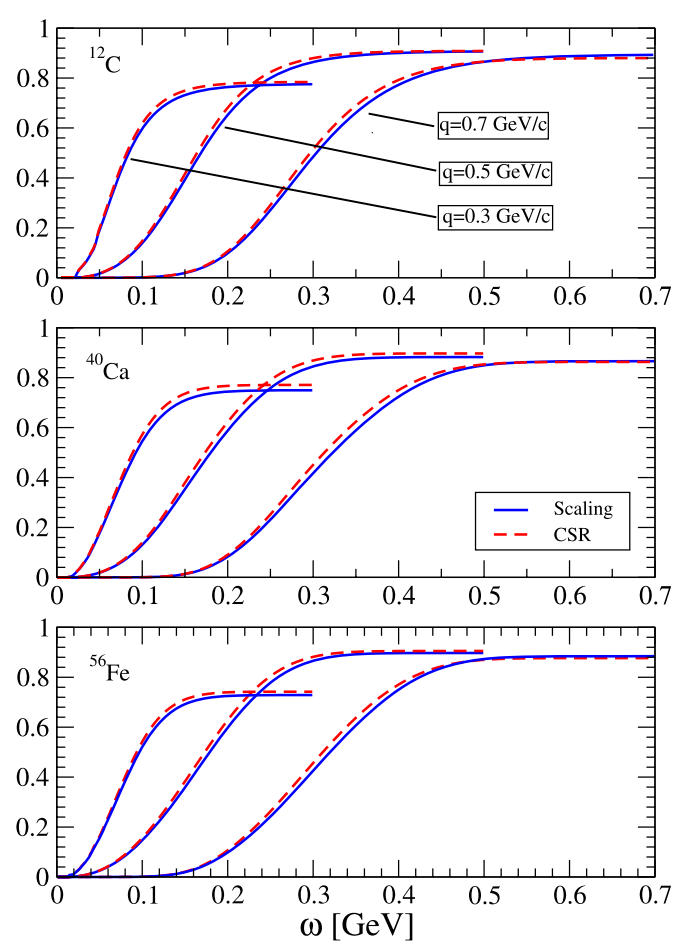

Fig. 2. (Color online.) Coulomb sum rule as a function of the energy transfer for ${ }^{12} \mathrm{C}$ (top panel), ${ }^{40} \mathrm{Ca}$ (middle) and ${ }^{56} \mathrm{Fe}$ (bottom). In each case, results obtained using the expression of the CSR given by Eq. (4) are compared with predictions based on the scaling analysis (6) for three different values of the momentum transfer.

with the effective electric form factor given by

$\widetilde{G}_{E}^{2}\left(Q^{2}\right)=\left[G_{E p}^{2}\left(Q^{2}\right)+\frac{N}{Z} G_{E n}^{2}\left(Q^{2}\right)\right] \frac{(1+\tau)}{(1+2 \tau)}$,

where $N$ and $Z$ are the neutron and proton numbers of the target, respectively, and $G_{E p}$ and $G_{E n}$ the Sachs electric form factors for proton and neutron. The term $\tau$ is the usual dimensionless quantity, $\tau \equiv\left|Q^{2}\right| / 4 M_{N}^{2}$ with $M_{N}$ the nucleon mass. The lower limit in the integration $\omega^{+}$includes all inelastic contributions but excludes the elastic peak.

An analog of the CSR can be also introduced in terms of the superscaled function and the scaling variable by taking into account the explicit expression of the longitudinal superscaling function, as well as its physics significance,

$\operatorname{CSR}_{\text {scal }}(q)=\int_{-\infty}^{\infty} d \psi^{\prime} f_{L}\left(\psi^{\prime}\right)$

Here the integration limits, denoted by $(-\infty,+\infty)$, extend in reality to the range allowed by kinematics and the experimental setup. Note that the scaling variable depends on the transferred momentum and energy, $q, \omega$. Expression (4) of the CSR used by experimentalists does not exactly correspond to Eq. (6) due to the fully relativistic expressions involved in the longitudinal scaling function [19] and to the different integration variable. Thus, in order to set down the impact of the particular CSR expression on the analysis of data, in what follows we compare results corresponding to Eqs. (4) and (6). The analysis is presented in Fig. 2 where we have considered three nuclei: ${ }^{12} \mathrm{C}$ (top panel), ${ }^{40} \mathrm{Ca}$ (middle) and ${ }^{56} \mathrm{Fe}$ (bottom). In each case we show how the CSR behaves as a function of the energy transfer $\omega$ for three different values of the momentum transfer $q$ : $0.3,0.5$ and $0.7 \mathrm{GeV} / c$. We compare the results corresponding to Eq. (4), denoted as CSR (dashed line), 

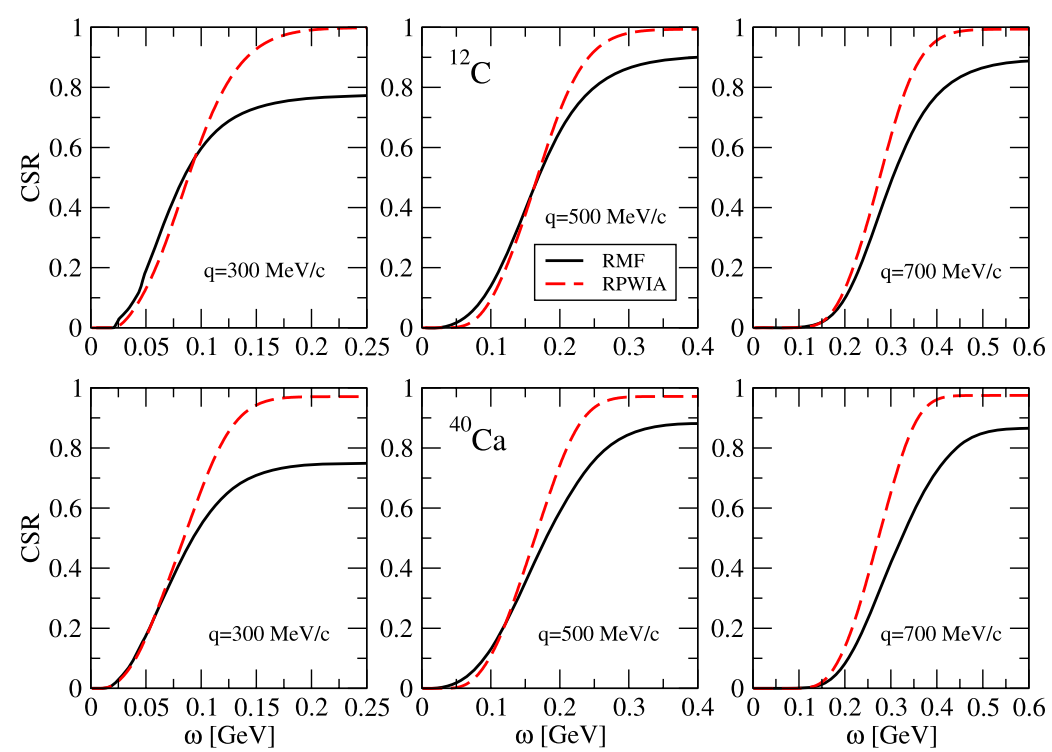

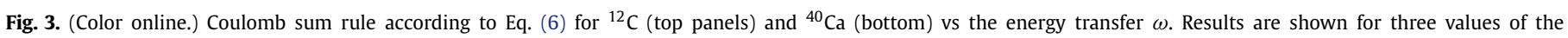
momentum transfer $q$, comparing RPWIA (dashed) and RMF (solid) approaches.

with the ones evaluated through the scaling function (6), denoted as scaling (solid line). We conclude that, apart from some minor discrepancies ascribed to the different single-nucleon expressions considered and the influence of the nuclear scale introduced in the longitudinal scaling function, both expressions for the CSR lead to similar results, hence drawing analogous conclusions. Notice that in all the cases the result given by Eq. (6) lies slightly below the one of (4) for intermediate values of the energy transfer. All results in Fig. 2 have been obtained with the RIA-RMF model.

Comparing the results obtained for the three nuclei, the CSR dependence with the target is seen to be very tiny. The CSR saturates to almost the same value for the three nuclei: $\sim 0.9$ for $q=0.5$ and $0.7 \mathrm{GeV} / c$ and $\sim 0.7$ for $q=0.3 \mathrm{GeV} / c$. Moreover, the behavior of the CSR is similar for the three targets, getting saturation, at each $q$-value, for very close transferred energies. Results in Fig. 2 allow us to focus on the CSR predictions given by Eq. (6) and to compare them to data arranged according to (4).

As shown in Fig. 1, the function $f_{L}^{\exp }\left(\psi^{\prime}\right)$ presents a long tail extended to large $\omega$-values, which is not reproduced by RPWIA and rROP calculations (neither by the majority of nonrelativistic models employed in the literature [21]). The presence of this important strength in $f_{L}^{\text {exp }}\left(\psi^{\prime}\right)$ may affect significantly the results for the CSR. Hence, in what follows we study how the CSR depends on the specific approach considered. To make easier the analysis we only consider two extreme cases: RPWIA, namely no FSI, and RMF, i.e., the presence of strong scalar and vector potentials in the final state. The shape of $f_{L}\left(\psi^{\prime}\right)$ in both cases is quite different, being the tail at large $\omega$-values largely absent in RPWIA.

Results are presented in Fig. 3 for ${ }^{12} \mathrm{C}$ (top panels) and ${ }^{40} \mathrm{Ca}$ (bottom) and three $q$ values: $q=300 \mathrm{MeV} / \mathrm{c}$ (left panels), $500 \mathrm{MeV} / c$ (middle) and $700 \mathrm{MeV} / c$ (right). One observes that the CSR saturates to $\sim 1$ for all $q$-values and the two nuclei in the case of RPWIA. On the contrary, the RMF description leads to a saturation value smaller than 1 , which grows with $q$ up to being of the order of 0.9 for $q \geqslant 0.5 \mathrm{GeV} / c$, i.e., where Pauli blocking is not in effect and thus also scaling holds. It is also important to point out that saturation is reached faster in RPWIA. This is consistent with the general symmetry shown by $f_{L}^{\mathrm{RPWIA}}\left(\psi^{\prime}\right)$ in contrast to the long tail presented by data and the RMF model (see Fig. 1). Part of the strength that has been shifted to high $\omega$-values in the RMF case (because of FSI) cannot be reached within the kinematical con-

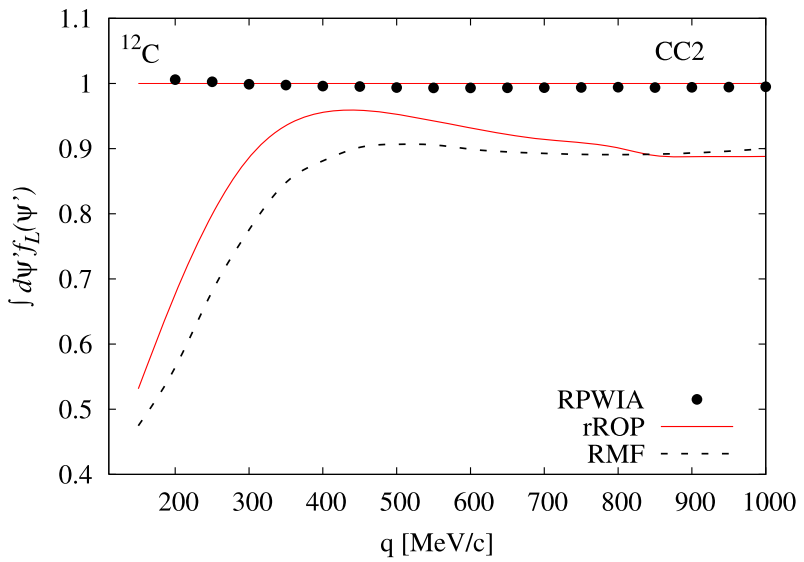

Fig. 4. (Color online.) Integrated scaling function versus the momentum transfer $q$. Results are shown for ${ }^{12} \mathrm{C}$ comparing three different models, integrated up to the maximum energy transfer allowed by kinematics (see text for details).

strains, hence leading to RMF-CSR results being smaller than the RPWIA ones. Comparing the results obtained for ${ }^{12} \mathrm{C}$ and ${ }^{40} \mathrm{Ca}$ as well as ${ }^{56} \mathrm{Fe}$ (not shown in the figure but following a very similar trend), the CSR dependence on the target is very tiny (see discussion in previous figure). This outcome is in accordance with second kind scaling property.

The behavior of the CSR with the momentum transfer $q$ is illustrated in Fig. 4, where we show the results for ${ }^{12} \mathrm{C}$ evaluated through Eq. (6) with different descriptions of the FSI: RPWIA, rROP and RMF, integrated up to the maximum energy transfer allowed by kinematics. The CC2 current operator has been considered, as results with this prescription agree fairly well with the superscaling function. As observed, RPWIA leads to unity for all $q$-values, even in the region where the CSR is not saturated in the RFG due to Pauli blocking, i.e., $q \leqslant 400-450 \mathrm{MeV} / c$. This is in contrast with the other two models with FSI turned on, which show a CSR that increases with $q$ up to becoming stable. Concerning specific CSR-saturated values, the rROP gets its maximum $(\sim 0.95)$ for $q=0.4 \mathrm{GeV} / c$, starting to diminish slightly in the region $0.4 \leqslant$ $q \leqslant 0.9 \mathrm{GeV} / c$ up to being of the order of $\sim 0.9$. The CSR result obtained within RMF increases with $q$ up to reaching $\sim 0.9$ 
Table 1

Integrated CSR evaluated within the RMF as a function of the momentum transfer $q$. Second column presents the maximum energy loss as indicated in Bates experiment [15]. We present the CSR results evaluated by extending the integration up to the maximum energy transfer allowed by kinematics (column 3 ) and up to the cutoff value used in Bates (column 4). Finally, column 5 reflects the difference between both results (percentage) and column 6 presents for reference the RFG predictions.

\begin{tabular}{llllcl}
\hline$q(\mathrm{MeV} / c)$ & $\omega_{\max }(\mathrm{MeV})$ & CSR (total) & CSR $\left(\omega_{\max }\right)$ & \% (diff.) & CSR $(\mathrm{RFG})$ \\
\hline 300 & 140 & 0.7493 & 0.6917 & 7.7 & 0.8197 \\
325 & 160 & 0.7889 & 0.7378 & 6.5 & 0.8640 \\
350 & 190 & 0.8207 & 0.7874 & 3.9 & 0.8975 \\
375 & 220 & 0.8458 & 0.8234 & 2.6 & 0.9289 \\
400 & 250 & 0.8638 & 0.8485 & 1.8 & 0.9483 \\
425 & 260 & 0.8759 & 0.8548 & 2.4 & 0.9649 \\
450 & 240 & 0.8822 & 0.8159 & 7.5 & 0.9683 \\
475 & 230 & 0.8842 & 0.7552 & 14.6 & 0.9707
\end{tabular}

Table 2

Same as Table 1, but for the kinematics considered at Saclay [12]

\begin{tabular}{lllll}
\hline$q(\mathrm{MeV} / c)$ & $\omega_{\max }(\mathrm{MeV})$ & CSR $($ total $)$ & CSR $\left(\omega_{\max }\right)$ & \% (diff.) \\
\hline 330 & 175 & 0.7889 & 0.7586 & 3.8 \\
370 & 195 & 0.8458 & 0.7953 & 6.0 \\
410 & 235 & 0.8638 & 0.8387 & 2.9 \\
450 & 265 & 0.8822 & 0.8490 & 3.8 \\
500 & 290 & 0.8825 & 0.8335 & 5.6 \\
550 & 310 & 0.8788 & 0.8003 & 8.9 \\
\hline
\end{tabular}

for $q \approx 400-500 \mathrm{MeV} / c$. This CSR value remains stabilized in the whole $q$ region explored in the figure, that is, up to $q=1 \mathrm{GeV} / c$. For higher $q$, not presented in Fig. 4, it can be shown that the CSR-RMF (likewise rROP) result starts to increase approaching 1 for $q \sim 1.6 \mathrm{GeV} / c$. However, for so large $q$-values, caution should be drawn on the assumptions implied by our theoretical description as well as by the extraction of the CSR from data. Finally, note that the strong potentials involved in the RMF, both for initial and final nucleon states, make the strength to be shifted to higher values of the (asymptotic) nucleon momentum [27].

Comparison between CSR theoretical results and experimental data requires to extract the Coulomb sum rule from the longitudinal response data by performing the integrals in Eqs. (4), (6) using as upper integration limits the specific $\omega$-cutoff values employed by the experimentalists. In particular, in the case of ${ }^{40} \mathrm{Ca}$, different experiments, Bates [15] and Saclay [12], have considered different $\omega_{\max }$-values as integration limits, as shown in Tables 1 and 2. One has to keep in mind that in the case of Bates data and for $q \geqslant 425 \mathrm{MeV} / c$, the value of the maximum energy transfer included in the experimental CSR diminishes as the momentum transfer $q$ goes up due to the uncertainties associated with the $\mathrm{L} / \mathrm{T}$ separation. This explains why in Table 1 , while the total CSR estimated under the RMF reaches a rather constant value $(\sim 0.88)$ for transferred momenta larger than $425 \mathrm{MeV} / c$, the predicted CSR under RMF employing the experimental energy transfer cutoff gets smaller for $q$ increasing, after $\sim 400 \mathrm{MeV} / c$.

In Fig. 5 we present results for ${ }^{40} \mathrm{Ca}$ corresponding to RMF (top panel) and RPWIA (bottom) approaches. In each case, three $q$-values have been considered, $q=300,400$ and $500 \mathrm{MeV} / c$. The CSR is shown as a function of the scaling variable $\psi^{\prime}(q, \omega)$. We also plot, for each $q$, the value of the scaling variable $\psi^{\prime}$ corresponding to the specific $\omega$-cutoffs given in the experimental papers. These span the regions: $140 \lesssim \omega \lesssim 150 \mathrm{MeV} / c$ for $q=300 \mathrm{MeV} / c, 230 \lesssim$ $\omega \lesssim 250 \mathrm{MeV} / c$ for $q=400 \mathrm{MeV} / c$, and $220 \lesssim \omega \lesssim 290 \mathrm{MeV} / c$ for $q=500 \mathrm{MeV} / c$. In the latter $(q=500 \mathrm{MeV} / c)$ the lower $\omega$-value represents the limit employed at Bates [15] and the larger one the cutoff included in Saclay [12]. These regions are presented as shadowed areas, where the color indicates the specific $q$-value which is
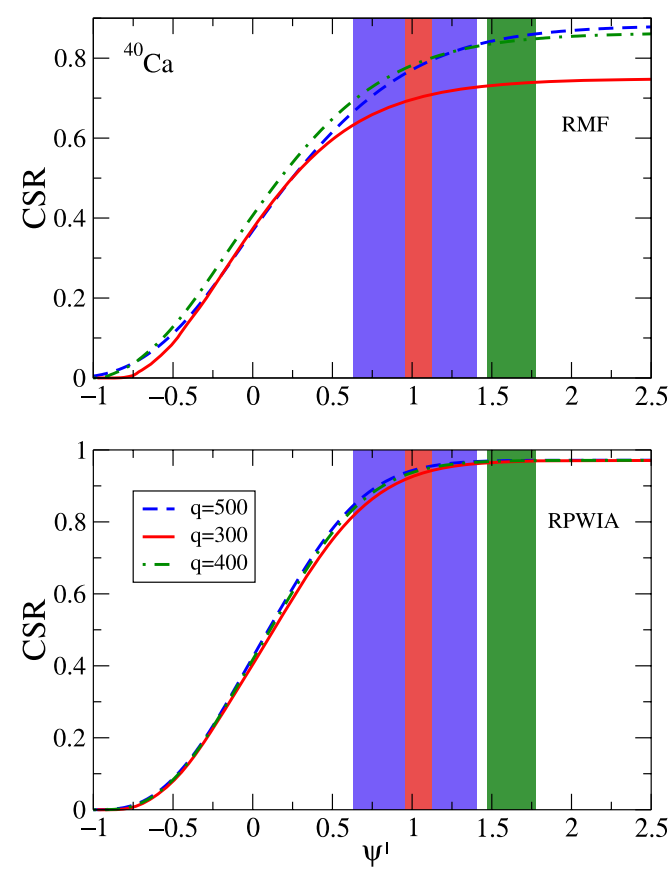

Fig. 5. (Color online.) Coulomb sum rule as a function of the scaling variable $\psi^{\prime}$ for ${ }^{40} \mathrm{Ca}$. Top panel refers to results obtained within the RMF approach and bottom to RPWIA. The vertical shadowed bands refer to the extreme values of $\psi^{\prime}$ corresponding to the energy transfer cutoffs considered in the analysis of the experiments. Each color refers to a different $q$-value, namely red $(q=300 \mathrm{MeV} / c)$, green $(q=400 \mathrm{MeV} / c)$ and blue $(q=500 \mathrm{MeV} / c)$. Lower limits in each band correspond to Bates values and higher ones to Saclay integration cutoff.

directly connected with the corresponding (same color) theoretical CSR result.

Results in Fig. 5 illustrate clearly the amount of saturation reached by the CSR at the maximum $\omega$-loss taken from the experiment. Let us consider the case $q=300 \mathrm{MeV} / c$ (solid red line and red shadowed band). Here the CSR saturates to $\sim 0.75$ for RMF and $\sim 0.97$ for RPWIA if the integration is extended over the whole allowed range (see Table 1). On the contrary, CSR results integrated up to the shadowed area are approximately $\sim 0.70$ (RMF) and $\sim 0.93$ (RWPIA). This means that saturation of CSR is reached at the order of $\sim 93 \%$ for RMF and $\sim 96 \%$ for RPWIA at the experimental energy cutoff. In other words, the $\omega$-values beyond the experimental accessible region correspond to $\mathrm{a} \sim 7 \%$ contribution to the fully integrated CSR in the RMF, and only $\sim 4 \%$ in the RPWIA case. These results reflect the increased tail of the longitudinal response in the RMF case. It is worth recalling, however, the different CSR values emerging from the two approaches, 0.75 in RMF and almost 1 (0.97) in RPWIA.

Similar comments apply also to higher $q$-values, $400 \mathrm{MeV} / \mathrm{c}$ (green color) and $500 \mathrm{MeV} / c$ (blue), although here the discrepancy between RMF and RPWIA results gets reduced because of the significant enhancement of the CSR value in the RMF approach. For $q=400 \mathrm{MeV} / c$, the RMF-CSR experimental cutoff result is on average $\sim 97 \%$ of the RMF-CSR for the whole range, whereas in RPWIA saturation is already reached at the experimental energy loss. Finally, in the case of $q=500 \mathrm{MeV} / c$ some comments apply because of the wide blue shadowed area linked to the very different $\omega$-cutoffs considered at Bates and Saclay, $\omega_{\max }=220 \mathrm{MeV}$ and $290 \mathrm{MeV}$, respectively. For the Saclay experiment [12], i.e., upper limit in the shadowed band, the CSR model evaluated up to the experimental cutoff includes $\sim 95 \%(\sim 100 \%)$ contribution of the total CSR strength in the RMF (RPWIA) approach. On the contrary, the contribution (integrated up to $\left.\omega_{\max }\right)$ reduces to $\sim 75 \%(\sim 95 \%)$ 
for RMF (RPWIA) in the case of the maximum energy loss used at Bates [15] (lower limit of the band, $\omega_{\max }=220 \mathrm{MeV}$ ). As it will be shown later on, this makes a significant difference when comparing theoretical calculations with the CSR extracted from both experiments.

It is important to point out again that the CSR obtained in the whole allowed $\omega$ range within the RMF approach saturates to $\sim 0.88$ for $q \geqslant 400,500 \mathrm{MeV} / c$, that is, $\sim 12 \%$ below the RPWIA result. Further the RMF-CSR result accumulated up to the experimental energy cutoff employed in Bates, is on average $~ 15-18 \%$ below the corresponding RPWIA result for $q=400,500 \mathrm{MeV} / c$, and $\sim 25 \%$ below for $q$ around $300 \mathrm{MeV} / c$. This is consistent with the behavior shown in Fig. 4. It is worth noticing that the contribution to the CSR of the strength outside the experimentally integrated region is different if considering RPWIA and/or RMF. In a model like RMF which agrees with the experimentally deduced longitudinal scaling response of Bates, the contribution of the unobserved tail beyond the cutoff employed at Bates is around $7 \%$ for $q \sim 300 \mathrm{MeV} / c, 2-3 \%$ around $q \sim 400 \mathrm{MeV} / \mathrm{c}$ and increases up to $15 \%$ for the largest $q$-value $(475 \mathrm{MeV} / c)$ measured at Bates.

To conclude, a comparison between theory and experimental data is provided in Fig. 6. First, in the left-top panel theoretical results for the CSR evaluated with the RMF approach applied to ${ }^{40} \mathrm{Ca}$ (red circles) and ${ }^{12} \mathrm{C}$ (green triangles) are presented. In both cases, CSR has been obtained making use of (6) and extending the upper integration limit to the maximum value permitted by the kinematics, i.e., once CSR has already reached saturation. Results in Fig. 6 show the independence of the CSR on the nuclear target, within the present approaches. For reference, we also include the CSR evaluated with the RFG model (black diamonds). Here, the CSR result approaches almost 1 for $q \sim 500 \mathrm{MeV} / c$, i.e., $q \geqslant 2 k_{F}$. For lower $q$-values Pauli-blocking effects are important giving rise to a significant reduction in the CSR value. Notice that, although the integral of $f_{L}^{R F G}$ (likewise the CSR) should be exactly one in the $\mathrm{QE}$ domain, the value in Fig. 6, slightly lower than 1 , reflects the shift energy included in the definition of the scaling variable $\psi^{\prime}$ (see $[19,24]$ for details). Theoretical results are compared with the Coulomb sum rule for ${ }^{40} \mathrm{Ca}$ extracted from data measured at Bates [15] for $q$-values in the domain, $300 \leqslant$ $q \leqslant 475 \mathrm{MeV} / c$. On general grounds, we observe that RMF results agree fairly well with data, lying slightly below for the smaller $q$-values, [300,350] $\mathrm{MeV} / c$, and above data for $q>400 \mathrm{MeV} / \mathrm{c}$ where the experimental uncertainty is significantly larger. Notice however, that the behavior shown by data, with a depletion occurring for $q \geqslant 400 \mathrm{MeV} / c$ is not reproduced by theoretical CSR calculations, which increase smoothly with $q$ approaching saturation. This discrepancy is mainly linked to the upper integration $\omega$-limits used in the analysis of data. Whereas theoretical CSR results were obtained through Eq. (6) extending the integral up to the maximum $\omega$, likewise $\psi^{\prime}$, value permitted by kinematics, Bates CSR data on the contrary, have been extracted making use of Eq. (4) with the upper $\omega_{\max }$ limit fixed, for each $q$, according to the values given in Table 2 of Ref. [15] (see also Table 1). In particular, notice the relatively low $\omega_{\max }$ values used by experimentalists for $q \sim 450,500 \mathrm{MeV} / c$. As we have already mentioned, significant strength in the CSR may be left out when using relatively low energy transfer cutoffs.

This is clearly illustrated in the right-top panel of Fig. 6, where we compare again Bates CSR data with RMF theoretical results, but these now evaluated using as upper integration limits the same $\omega_{\max }$ values considered in the experiment (see Table 1 in this work and Table 2 in [15]). Compared with previous results, a decrease in the RMF-CSR is observed, depending its magnitude on the specific momentum transfer considered: from $\sim 2-4 \%$ for the central $q([350-425] \mathrm{MeV} / c)$ and $\sim 6-8 \%$ for $q=300,325,450 \mathrm{MeV} / c$, up to $\sim 15 \%$ for $q=475 \mathrm{MeV} / c$. This explains the depletion presented by the CSR (theory and data) for larger $q$. Concerning the comparison between theory and data, we observe that Bates CSR data are reproduced within the RMF approach. Only for $q=300$ and $325 \mathrm{MeV} / c$, RMF results underestimate data by $\sim 10-12 \%$. This discrepancy can be partly ascribed to the different expressions used to evaluate the CSR, Eq. (4) for experimental data and (6) for RMF. As shown in Fig. 2, using (4) and/or (6) lead to slightly different CSR results, being the former larger for the $\omega_{\max }$-values considered in the experiment. Hence, the discrepancy between theory and experiment in Fig. 6 reduces by $\sim 3 \%-5 \%$ when Eq. (4) is also considered within the RMF approach. Further, for the lowest values of momentum transfer, discrete inelastic excitations of the nuclei may be present in the data, while they are not considered in the purely QE nucleon knockout estimations of the models. From this analysis, we conclude that our theoretical model describes quite consistently Bates data, with a minor underestimation (within the experimental error bars), indicating that no additional quenching of the relativistic models is needed, other than the $\sim 10-15 \%$ strength that is shifted outside the experimentally available region for the $L$ channel. This is consistent with the good agreement found between the RMF model and the experimental longitudinal scaling function.

The previous argument is also reinforced by results shown in the bottom panels of Fig. 6. Here we present the ratio of integrated response functions to the longitudinal strength calculated from the RFG model. We compare data from Bates experiment (blue squares) with those given by Meziani et al. [12] (green triangles) and the theoretical results evaluated within the RMF approach (red circles). As in the previous discussion, the left-bottom panel refers to RMF results evaluated by extending the integral (6) up to the whole region allowed by kinematics, and dividing by the RFG results. This explains why the RMF approach leads to very similar values $(\sim 0.9)$ for all $q$, as the comparison of RMF to RFG results is rather constant with $q$ if integration includes the whole tail region. On the contrary, in the right-bottom panel, theoretical RMF-CSR results have been evaluated by using the specific $\omega_{\max }$-limits considered at Bates [15] for each momentum transfer (red circles). We also show the RMF results obtained by using the momentum transfers $q$ and energy losses $\omega_{\max }$ given by Meziani et al. [12] corresponding to Saclay experiment (black diamonds). Apart from the slightly different $q$ values used in Bates and Saclay, the difference between the RMF results corresponding to both experimental setups comes from the energy transfer cutoffs considered (see Tables 1 and 2). The effect of the cutoff is particularly visible for $q \sim 475-500 \mathrm{MeV} / c$ where the larger $\omega_{\text {max }}{ }^{-}$ values considered in Saclay lead to higher RMF-CSR results, making the theoretical prediction to depart even further from data. Therefore, from the general analysis shown in Fig. 6, we observe that RMF calculations are compatible with Bates data in the whole $q$ region, apart from some deviation (underpredicting data) for the lowest $q=300,325 \mathrm{MeV} / c$. On the contrary, data from Saclay experiment show an important depletion ( $\gtrsim 40 \%$ ) with regards to the theoretical RMF predictions, even when these data should include in principle more contribution from the high energy tail (compared with Bates). This depletion is not present in Bates data and is neither supported by our theoretical estimates. According to the analysis carried out in this work, this difference in behaviour of Saclay and Bates data cannot be due to strength outside the experimental bounds for the energy transfer.

\section{Conclusions}

The study of the CSR and its extraction from the analysis of the separated $L$ contribution to QE electron scattering data has been 

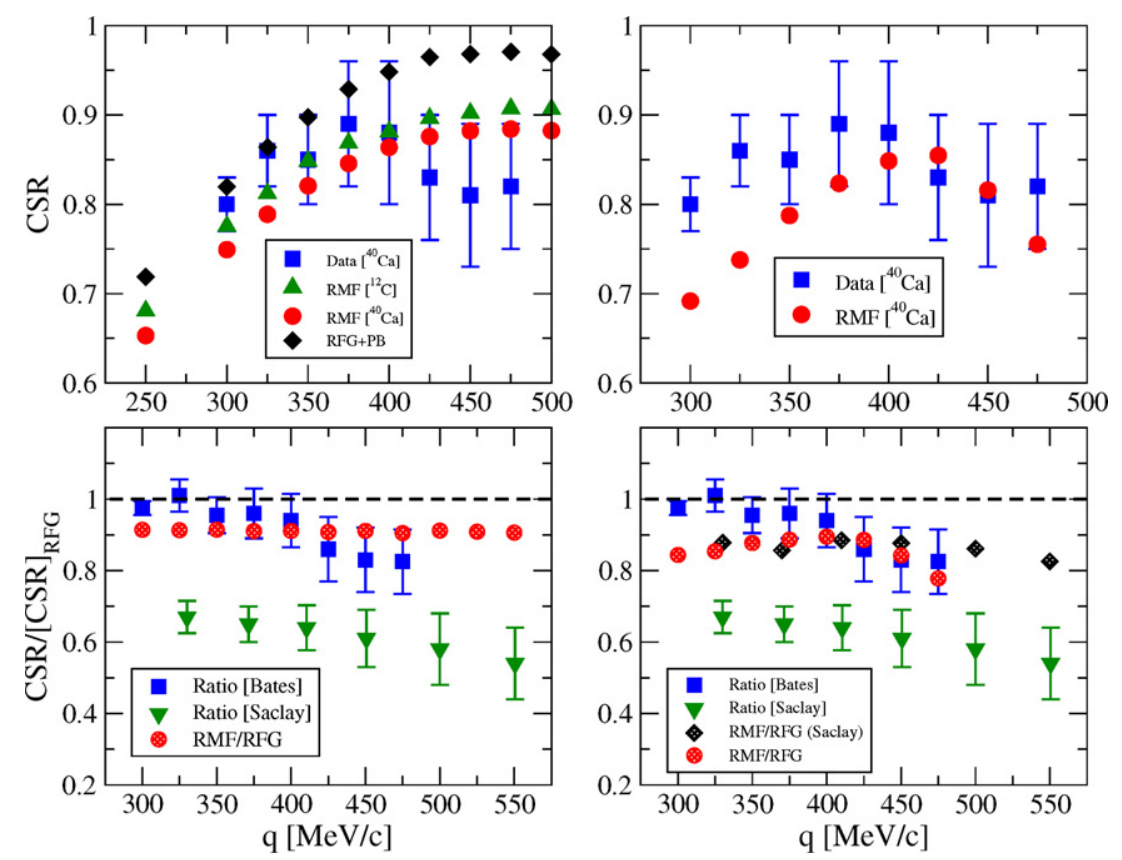

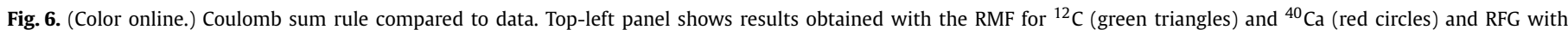

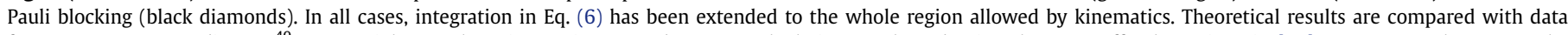

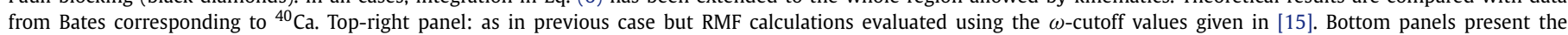
ratio between RMF results and RFG ones compared to data from Bates and Saclay (see text for details).

extensively reviewed by different authors leading to rather controversial results. This controversy is directly linked to the interpretation of experiments as well as to the theoretical descriptions and the role played by different ingredients. Whereas in some works it is concluded that a significative quenching occurs in the observed CSR, others show that only a very mild reduction (or no reduction at all) is observed from the analysis of data. Being aware that new, high precision, data expected from JLAB at high energy transfer would help in disentangling between different approaches, in this work we try to shed some light on this problem analyzing also its connection with the general scaling properties observed by inclusive $\mathrm{QE}$ electron scattering. Scaling arguments applied to $\left(e, e^{\prime}\right)$ data have clearly proved to high accuracy how well scaling is respected by QE data. Moreover, a "universal" superscaling function has been extracted from the analysis of separated longitudinal data, showing a representative shape with a long tail that extends to high values of the energy transfer. As we have shown, this extended tail, with regards to usual nonrelativistic models or plane wave approaches, must be kept in mind if making estimates of the contribution to the CSR coming from outside the experimentally explored region.

A careful analysis has been performed using different theoretical approaches: RPWIA, RMF, rROP. Results have shown that the CSR is basically independent on the nuclear system considered. Obviously, for heavy nuclei Coulomb distortion of the electron wave functions would need to be taken into account in order to extract reliable longitudinal response from the data, but this will not likely affect the theoretical estimations made in this work. Concerning how the Coulomb sum rule reaches its saturation value, we have observed that RPWIA gets saturation faster than RMF. This is in accordance with the general shape shown by the superscaling function in both cases, being the tail for large $\omega$-values absent in RPWIA. Furthermore, whereas RPWIA leads to a saturated CSR very close to 1 for all $q$-values, even when the integration is limited to the range experimentally considered, the RMFCSR integrated in the whole allowed range gets about $\sim 0.87$ for $q \geqslant 0.4-0.5 \mathrm{GeV} / c$, and this value keeps stabilized for $q$ up to the maximum $q \sim 1 \mathrm{GeV} / c$ explored in this work.

In order to compare our theoretical predictions with experiment, we have analyzed the role played by the cutoff $\omega$-value considered as upper integration limit in the expression of the CSR. From our results, we conclude that the Coulomb sum rule from RMF reaches $\sim 85-95 \%$ of its saturated value if truncation at the experimental $\omega$-cutoff is taken. The largest strength lost in CSR occurs for $q=475 \mathrm{MeV} / c$, and is of the order of $\sim 15 \%$. The comparison with CSR results obtained from data measured at Bates for ${ }^{40} \mathrm{Ca}$ has shown its accordance with the RMF approach. Similar comments apply to the ratio of integrated response functions to the $L$ strength evaluated with the RFG. These results show that no further quenching than the one predicted in the relativistic mean field impulse approximation is needed to describe the longitudinal response measured at BATES, that shows a depletion of the free value of the order of $\sim 10-20 \%$. This is in contrast with data measured at Saclay showing a reduction of the $L$ channel of the order of $\sim 30-40 \%$ [12-14], in spite of the fact that in these experiments the cutoff in the energy transfer is larger than for Bates experiments. The reasons for this difference would hopefully be clarified by the recent experiment at JLAB [16].

\section{Acknowledgements}

This work was partially supported by DGI (MICINN-Spain) contracts FIS2008-04189, FPA2007-62216, by the UCM and Comunidad de Madrid (Grupo de Física Nuclear, 910059), the Spanish Consolider-Ingenio programme CPAN (CSD2007-00042), by the Junta de Andalucía, and by the INFN-CICYT Collaboration agreements INFN08-20 \& FPA2008-03770-E/INFN. This work has benefited from discussions with M.B. Barbaro and T.W. Donnelly.

\section{References}

[1] S. Frullani, J. Mougey, Adv. Nucl. Phys. 14 (1984) 1. 
[2] S. Boffi, C. Giusti, F.D. Pacati, M. Radici, Phys. Rep. 226 (1993) 1; S. Boffi, C. Giusti, F.D. Pacati, M. Radici, Electromagnetic Response of Atomic Nuclei, Oxford University Press, Oxford, 1996.

[3] J.J. Kelly, Adv. Nucl. Phys. 23 (1996) 75.

[4] A.S. Raskin, T.W. Donnelly, Ann. of Phys. 191 (1989) 78; T.W. Donnelly, A.S. Raskin, Ann. of Phys. 169 (1986) 247.

[5] J.D. Walecka, Electron Scattering for Nuclear and Nucleon Structure, Cambridge Univ. Press, 2001.

[6] C.R. Chinn, A. Picklesimer, J.W. Van Orden, Phys. Rev. C 40 (1989) 790.

[7] J. Jourdan, Nucl. Phys. A 603 (1996) 117.

[8] J.M. Udías, P. Sarriguren, E. Moya de Guerra, E. Garrido, J.A. Caballero, Phys. Rev. C 48 (1993) 2731; J.M. Udías, P. Sarriguren, E. Moya de Guerra, E. Garrido, J.A. Caballero, Phys. Rev. C 51 (1995) 3246.

[9] J.M. Udías, P. Sarriguren, E. Moya de Guerra, J.A. Caballero, Phys. Rev. C 53 (1996) R1488.

[10] J.M. Udías, J.A. Caballero, E. Moya de Guerra, J.R. Vignote, A. Escuderos, Phys Rev. C 64 (2001) 024614

[11] K.V. McVoy, L. Van Hove, Phys. Rev. 125 (1962) 1159

[12] Z.E. Meziani, et al., Phys. Rev. Lett. 52 (1984) 2130

[13] Z.E. Meziani, et al., Phys. Rev. Lett. 69 (1992) 41.

[14] J. Morgenstern, Z.E. Meziani, Phys. Lett. B 515 (2001) 269.

[15] C.F. Williamson, et al., Phys. Rev. C 56 (1997) 3152.

[16] Jian-Ping Chen, Seonho Choi, Zein-Eddine Meziani, Spokesperson, JLAB E-05110, http://hallaweb.jlab.org/experiment/E05-110/exp_home, Precision Measurement of Longitudinal and Transverse Response Functions of Quasi-Elastic Scattering in the Momentum Transfer Range $0.55 \mathrm{GeV} / c<q<0.9 \mathrm{GeV} / c$.

[17] D.B. Day, J.S. McCarthy, T.W. Donnelly, I. Sick , Annu. Rev. Nucl. Part. Sci. 40
(1990) 357

[18] T.W. Donnelly, I. Sick, Phys. Rev. Lett. 82 (1999) 3212; T.W. Donnelly, I. Sick, Phys. Rev. C 60 (1999) 065502.

[19] C. Maieron, T.W. Donnelly, I. Sick, Phys. Rev. C 65 (2002) 025502.

[20] W.M. Alberico, A. Molinari, T.W. Donnelly, E.L. Kronenberg, J.W. Van Orden, Phys. Rev. C 38 (1988) 1801.

[21] J.E. Amaro, M.B. Barbaro, J.A. Caballero, T.W. Donnelly, C. Maieron, Phys. Rev. C 71 (2005) 065501.

[22] M.B. Barbaro, R. Cenni, A. De Pace, T.W. Donnelly, A. Molinari, Nucl. Phys. A 643 (1998) 137.

[23] J.A. Caballero, J.E. Amaro, M.B. Barbaro, T.W. Donnelly, C. Maieron, J.M. Udías, Phys. Rev. Lett. 95 (2005) 252502

[24] J.A. Caballero, Phys. Rev. C 74 (2006) 054603.

[25] C. Maieron, M.C. Martínez, J.A. Caballero, J.M. Udías, Phys. Rev. C 68 (2003) 048501.

[26] E.D. Cooper, S. Hama, B.C. Clark, R.L. Mercer, Phys. Rev. C 47 (1993) 297.

[27] J.E. Amaro, M.B. Barbaro, J.A. Caballero, T.W. Donnelly, J.M. Udías, Phys. Rev. C 75 (2007) 034613.

[28] J.A. Caballero, J.E. Amaro, M.B. Barbaro, T.W. Donnelly, J.M. Udías, Phys. Lett. B 653 (2007) 366

[29] O. Benhar, V.R. Pandharipande, Steven C. Pieper, Rev. Mod. Phys. 65 (1993) 817.

[30] O. Benhar, D. Day, I. Sick, Rev. Mod. Phys. 80 (2008) 189.

[31] O. Benhar, Nucl. Phys. A 476 (1988) 272.

[32] M.B. Barbaro, R. Cenni, T.W. Donnelly, A. Molinari, Phys. Rev. C 78 (2008) 024602.

[33] A. Meucci, J.A. Caballero, C. Giusti, F.D. Pacati, J.M. Udías, Phys. Rev. C 80 (2009) 024605.

[34] T. de Forest, Nucl. Phys. A 392 (1983) 232. 